\title{
Aberrant prefrontal-parietal-cerebellar circuits in alcohol dependence
}

This article was published in the following Dove Press journal:

Neuropsychiatric Disease and Treatment

\author{
Run Liu',* \\ $\mathrm{Bi}-\mathrm{Xia} \mathrm{Liu}{ }^{2, *}$ \\ Mingyue $\mathrm{Ma}^{1, *}$ \\ Dan Kong ${ }^{3, *}$ \\ Guanglin $\mathrm{Li}^{4}$ \\ Junle Yang' \\ Xiaoping $\mathrm{Wu}^{\prime}$ \\ Jiyong Zheng ${ }^{3}$ \\ Yan Dong'
}

'Department of Radiology, The Affiliated Xi'an Central Hospital of Xi'an Jiaotong University, Xi'an, 7I0004 Shaanxi, People's Republic of China; ${ }^{2}$ Department of ICU, Jiangxi Provincial Cancer Hospital, Nanchang, 330029 Jiangxi, People's Republic of China; ${ }^{3}$ Department of Medical Imaging, The Affiliated Huaian No. I People's Hospital of Nanjing Medical University, Huaian, 223300 Jiangsu, People's Republic of China; ${ }^{4}$ Honghui Hospital, Xi'an Jiaotong University, Xi'an, 7I0054 Shaanxi, People's Republic of China

*These authors contributed equally to this work

Correspondence: Jiyong Zheng Department of Medical Imaging, The Affiliated Huaian No. I People's Hospital of Nanjing Medical University, 6 Beijing Road West, Huaian, Jiangsu 223300, People's Republic of China Email jyzhengdoctor@126.com

Yan Dong

Department of Radiology, The Affiliated Xi'an Central Hospital of Xi'an Jiaotong University, No. I6I, Xiwu Road, Xi'an, 710004 Shaanxi, People's Republic of China

Email shuijingbeier@।26.com
Objective: To identify whether the amplitude of low-frequency fluctuations (ALFF) analysis has the potential to serve as a biological marker to detect alcohol-induced spontaneous brain activities and distinguish the patients with alcohol dependence from the healthy subjects.

Methods: We utilized the ALFF analysis to report on the alcohol-induced spontaneous brain activities in 29 patients with alcohol dependence ( 9 female, 20 male) relative to 29 status-matched healthy subjects ( 11 female, 18 male). Receiver operating characteristic curve was used to test the ability of the ALFF analysis in discriminating the patients with alcohol dependence from the healthy subjects. Pearson correlation was used to evaluate the relationships between the signal value of those ALFF differences in brain areas and behavioral characteristics.

Results: Alcohol-induced brain differences located in the right inferior parietal lobule and right supplementary motor area with significant higher ALFF values, and in the left precuneus and bilateral cerebellum posterior lobe with lower ALFF values. The movement-related areas were significantly correlated with each other $(P<0.05)$. Receiver operating characteristic curve revealed good area under the curve values (mean, $0.86 \pm 0.079 ; 0.774-0.951$ ) of the ALFF differences in those specific brain areas, as well as high degree of sensitivities (mean, $80.84 \% \pm 14.01 \%$ or $80 \% \pm 14.56 \% ; 62.5 \%-100 \%$ ) and specificities (mean, $83.32 \% \pm 9.31 \% ; 70.8 \%-95.8 \%$ or $84.16 \% \pm 8 \% ; 75 \%-95.8 \%)$.

Conclusion: The ALFF analysis may serve as a biological indicator to detect the spontaneous brain activities in patients with alcohol dependence. The prefrontal-parietal-cerebellar circuit appears to be disturbed by long-term alcoholism in patients with alcohol dependence.

Keywords: alcohol dependence, amplitude of low-frequency fluctuations, receiver operating characteristic

\section{Introduction}

Alcohol consumption, the most frequent substance addiction with a high morbidity or mortality, is a serious public problem. The pernicious effects of extravagant alcohol consumption on brain and behavior are well known. It brings numerous adverse health consequences such as esophageal/liver cancer, liver cirrhosis, and vehicle accidents. ${ }^{1}$ Alcohol consumption would bring perceived relief from the negative emotions, such as stress and anxiety, which may thereby increase and/or reinforce the likelihood of future drinking behavior. ${ }^{2}$ Therefore, alcohol dependence is a chronic relapsing disorder, characterized by morbid alcohol consumption.

Modern imaging methods promote scholars to investigate the neurobiology mechanisms and consequences caused by alcohol addiction. These imaging methods are widely accepted for the detection of specific regional brain alterations in diseases. Resting-state functional MRI (rs-fMRI) can be used to visualize the brain activities associated with oxygenation and blood flow changes, and the responses of regional 
brain blood flow to drug-related cues. Furthermore, the rs-fMRI also can be used to visualize the addictive symptoms and cognitive capacity caused by alcohol consumption. These changes cannot be identified by traditional fMRI analyses. Therefore, the rs-fMRI is suitable to detect the alteration of spontaneous neuronal brain activity and can be used for pathophysiological mechanism exploration for several diseases. ${ }^{3}$ With the rapid development of these rsfMRI technologies, neuroimaging studies have described diverse pernicious effects from alcohol dependence, including the neurochemical changes and regional functional activity in the brain, ${ }^{4}$ which may yielded insights into the neurobiological mechanisms underlying alcohol dependence and may help clinicians assess the disease and aid in dynamic monitoring of the response to therapy, as well as guide care interventions. Alcohol dependence is associated with the changes of regional brain activities in several areas, which makes its neurobiological mechanism more complex. Although recent studies of structural and functional studies have increased tremendously and have identified several brain regions that are relevant to alcoholism, ${ }^{5,6}$ the neurobiological mechanism underlying alcohol dependence remains largely unknown.

The amplitude of low-frequency fluctuations (ALFF) analysis does not require prior hypothesis and/or knowledge, and has good test-retest reliability, which makes it useful for location of altered brain regions with abnormal spontaneous neuronal brain activity., ${ }^{3,-10}$ Therefore, the reliable characterization and simple calculation make the ALFF analysis a potential useful tool to study the alcohol dependence-induced intrinsic brain activities. ${ }^{4}$ Recently, the use of the ALFF analysis has been successfully applied in several disorders. ${ }^{3,11-13}$ However, to our knowledge, it has not been used in alcohol dependence. In the current study, we hypothesized that the alcohol dependence is associated with distinct intrinsic neuronal spontaneous activity in several brain areas with ALFF changes. To test the hypothesis, the current study utilized the ALFF analysis to identify altered functional brain areas in 29 patients with alcohol dependence relative to 29 status-matched healthy subjects. Next, we used Pearson correlations to evaluate the relationships between those brain areas with ALFF differences and behavioral characteristics. We also utilized receiver operating characteristic (ROC) curve to investigate whether the regional brain areas with ALFF differences have the ability to distinguish the patients with alcohol dependence from the status-matched healthy subjects.

\section{Materials and methods Subjects}

A total of 29 patients with alcohol dependence ( 9 female, 20 male; education, $9.52 \pm 2.87$ years; age, $48.62 \pm 6.81$ years; mean $\pm \mathrm{SD})$ and 29 status-matched healthy subjects (11 female, 18 male; education, $8.48 \pm 3.1$ years; age, $48.48 \pm 7.05$ years) participated in the present study. The life history of psychiatric disorders, daily alcohol consumption, Severity of Alcohol Dependence Questionnaire (SADQ), mean years of alcohol consumption, and Alcohol Use Disorders Identification Test (AUDIT) were recorded by an experienced psychiatrist who had worked for more than 10 years.

Patients with alcohol dependence met the diagnostic criteria as defined by The Diagnostic and Statistical Manual of Mental Disorders, version IV (DSM-IV). All recruited volunteers met the following inclusion criteria as in previous studies, ${ }^{14,15}$ firsttime visitors who had not taken any medications treatment before, had no history of other substance dependence or abuse, had no sleep disorders and major psychiatric disorders, had no foreign implants, and had no pathological brain MRI findings. The present study was approved by the Medical Research Ethical Committee of The Affiliated Huai' an No. 1 People's Hospital of Nanjing Medical University in accordance with the Declaration of Helsinki. The written informed consent from all volunteers was collected.

\section{MRI parameters}

We performed the MRI scan on a 3.0 Tesla MR scanner (Siemens, Munich, Germany). First, 176 high-resolution 3D T1-weighted anatomical images of in sagittal orientation (repetition time $/$ echo time $=1,950 / 2.3 \mathrm{~ms}$, gap $/$ thickness $=0 / 1 \mathrm{~mm}$, field of view $=244 \times 252 \mathrm{~mm}^{2}$, acquisition matrix $=248 \times 256$, flip angle $=9^{\circ}$ ) were collected. Next, we collected 240 functional images (repetition time/echo time $=3,000 / 25 \mathrm{~ms}$, gap/thickness $=0.5 / 5.0 \mathrm{~mm}$, flip angle $=90^{\circ}$, acquisition matrix $=32 \times 32$, field of view $=210 \times 210 \mathrm{~mm}^{2}$ ) covering the whole brain.

\section{Data analysis}

First, we deleted the first 10 time points of the functional images. The remaining data were dealt with standards for form transformation. The data preprocessing of the remaining data was made up of the following steps: including the slice timing, head motion correction, spatial normalization to the Montreal Neurological Institute space, and smoothing with Gaussian kernel of $6 \times 6 \times 6 \mathrm{~mm}^{3}$. The data of volunteers with $>1.5 \mathrm{~mm}$ maximum translation or/and $>1.5$ degree of rotation in any 
direction were discarded. After this, the remaining images were resampled at a resolution of $3 \times 3 \times 3 \mathrm{~mm}^{3}$ during the step of spatial normalization. Linear regression was applied to remove the effects of spurious covariates, including the Friston 24 head motion parameters, white matter signal, and cerebrospinal fluid signal. Next, the functional images were entered into temporal bandpass filter $(0.01-0.1 \mathrm{~Hz})$ and linearly detrended. The calculation details of the ALFF analysis have been presented in previous studies. ${ }^{3,10,16}$

\section{ROC curve and brain-behavior correlation analysis}

Recently, ROC curve has been increasingly applied to identify whether one image analysis may serve as a biological indicator to distinguish one group from another group. ${ }^{4,11,14}$ The mean $\beta$ values of the ALFF differences were extracted for ROC curve to identify whether the ALFF analysis may server as a biological indicator to distinguish the patients with alcohol dependence from healthy subjects. Pearson correlation analysis was used to evaluate the relationships between the ALFF differences in brain areas and behavioral characteristics. The statistical threshold was set at $P<0.05$.

\section{Statistical analysis}

Data are presented as mean \pm SD. The demographic characteristics (age, AUDIT score, and years of education) were analyzed with independent sample unpaired $t$-tests. The categorical data (sex distribution) were compared using Chi-squared $\left(\chi^{2}\right)$ test. The statistical threshold was set at $P<0.05$.

Before comparing the ALFF differences between groups, we used one-sample $t$-tests to construct within-group statistical maps of ALFF analysis to identify the network distributions of each group $(P<0.001$, false discovery rate (FDR) corrected). Then, independent sample unpaired $t$-tests were utilized to study the ALFF differences in regional brain areas between patients with alcohol dependence and healthy controls with nuisance covariates (age, sex, and years of education) of no interest. AlphaSim correction (threshold of individual voxel of $P<0.01$, cluster level of $P<0.05$ with contiguous voxel volume $\geq 1,620 \mathrm{~mm}^{3}$ ) was used to determine the statistical differences.

\section{Results}

\section{Sample characteristics}

The behavioral characteristics of the alcohol dependence and the healthy subjects are presented in Table 1. Patients with alcohol dependence did not significantly differ from healthy subjects in mean age $(t=0.076, P=0.94)$, sex distribution $\left(\chi^{2}=0.305, P=0.581\right)$, and mean education $(t=1.318$, $P=0.193)$. The mean AUDIT score was higher in patients with alcohol dependence than healthy controls $(t=20.353$, $P<0.001)$. In the alcohol dependence group, the mean duration of drink history was $(27.93 \pm 10.28$ years, range: $7-45$ years), the mean SADQ score was (20.34 \pm 6.89$)$, and the mean daily alcohol consumption was (mean $\pm \mathrm{SD}$, $239.66 \pm 107.22 \mathrm{~mL}$ ).

\section{ALFF differences}

Before comparing the ALFF differences between patients with alcohol dependence and healthy controls, one-sample $t$-test were used to construct within-group statistical maps for alcohol dependence group (Figure 1A) and healthy subjects (Figure 1B) separately ( $P<0.001$, FDR corrected). We found that the locations of the ALFF differences in brain areas of patients with alcohol dependence differ from healthy subjects in several areas (Figure 1A and B). Next, we performed $t$-test to investigate the ALFF differences between groups. Patients with alcohol dependence relative to healthy subjects demonstrated differences in brain areas in the right inferior parietal lobule (Brodmann's area [BA] 40)

Table I Characteristics of alcohol dependents and healthy subjects

\begin{tabular}{l|l|l|l|l}
\hline Demographics & Alcohol dependents & Healthy subjects & $\boldsymbol{t} / \chi^{2}$ value & $P$-value \\
\hline Mean age, years & $48.62 \pm 6.8 I$ & $48.48 \pm 7.05$ & 0.076 & 0.94 \\
Sex (male, female) & $29(20,9)$ & $29(18,1 \mathrm{I})$ & $0.305^{\mathrm{a}}$ & $0.58 \mathrm{I}$ \\
Education, years & $9.52 \pm 2.87$ & $8.48 \pm 3.1$ & 1.318 & 0.193 \\
Years of alcohol consumption & $27.93 \pm 10.28$ & $\mathrm{~N} / \mathrm{A}$ & $\mathrm{N} / \mathrm{A}$ & $\mathrm{N} / \mathrm{A}$ \\
SADQ score & $20.34 \pm 6.89$ & $\mathrm{~N} / \mathrm{A}$ & $\mathrm{N} / \mathrm{A}$ & $\mathrm{N} / \mathrm{A}$ \\
AUDIT score & $23.83 \pm 5.55$ & $2.55 \pm 0.95$ & 20.353 & $<0.001$ \\
Daily alcohol consumption, $\mathrm{mL}$ & $239.66 \pm 107.22$ & $\mathrm{~N} / \mathrm{A}$ & $\mathrm{N} / \mathrm{A}$ & $\mathrm{N} / \mathrm{A}$ \\
\hline
\end{tabular}

Notes: ${ }^{\mathrm{a}} \chi^{2}$ test; data are mean $\pm \mathrm{SD}$.

Abbreviations: AUDIT, Alcohol Use Disorders Identification Test; N/A, not applicable; SADQ, Severity of Alcohol Dependence Questionnaire. 
A

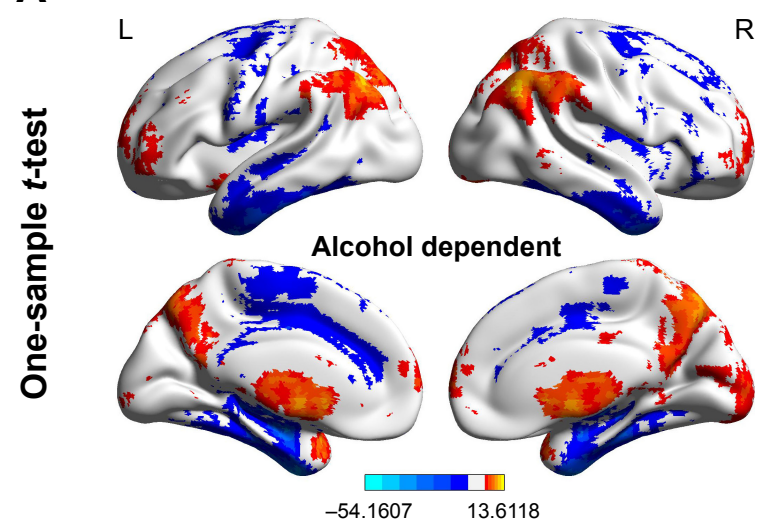

C

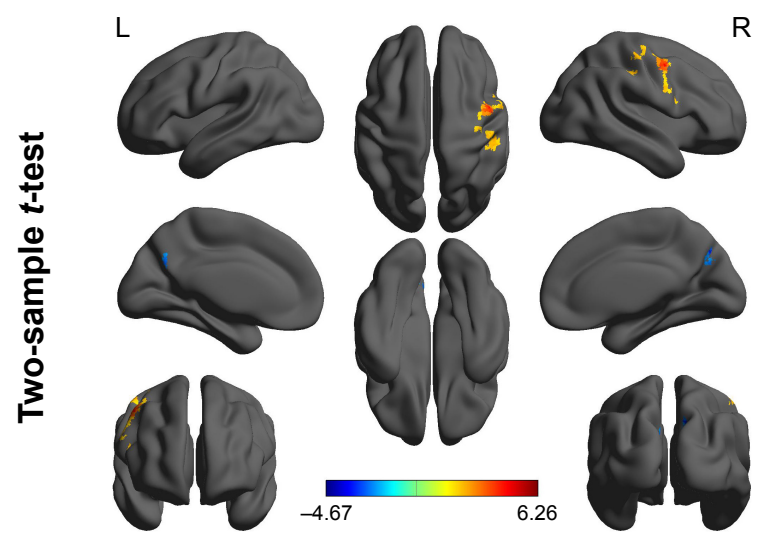

B

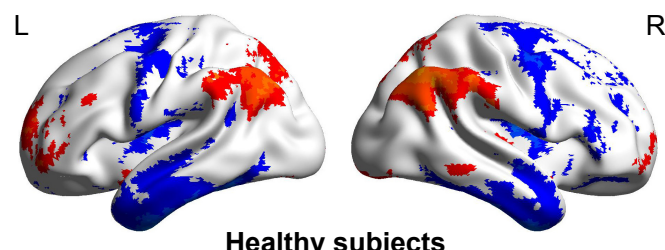

Healthy subjects

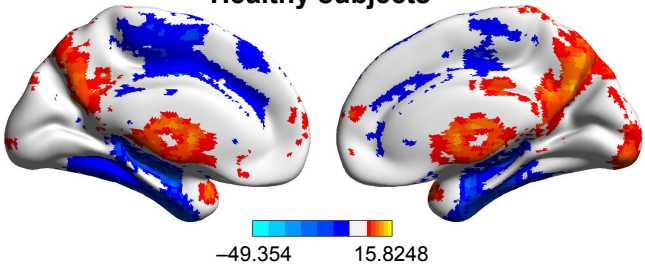

D

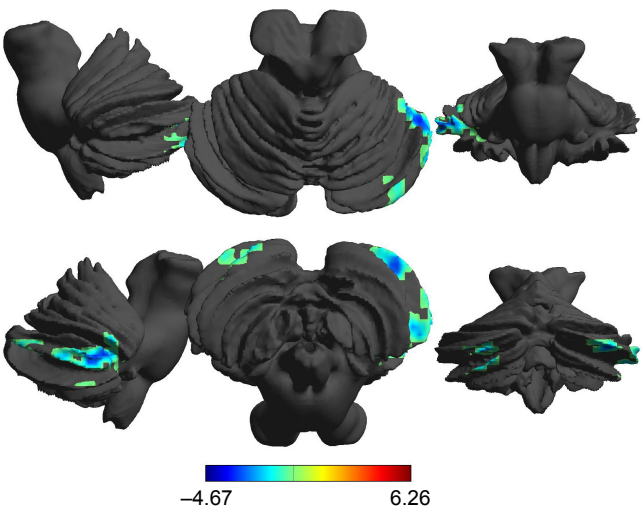

Figure I Altered ALFF areas in patient with alcohol dependence relative to healthy subject.

Notes: Results of one-sample $t$-test (A, B) and two-sample $t$-test (C, D). Red color, increased ALFF areas; blue color, decreased ALFF areas.

Abbreviations: ALFF, amplitude of low-frequency fluctuation; L, left; R, right.

and right supplementary motor area (BA 6) with higher ALFF values, and the left precuneus (BA 7) and bilateral cerebellum posterior lobe with lower ALFF values (Table 2, Figure $1 \mathrm{C}$ and D).

\section{ROC curve}

The mean $\beta$ value of those altered ALFF values in brain areas was extracted (Figure 2) for ROC curve analysis. The area under the curve (AUC) value is considered as good to excellent if it is $>0.8$, fair if between 0.7 and 0.8 , and poor to failed if lower than 0.7. ${ }^{17}$ Our findings demonstrated that the ROC curve revealed good AUC values (mean $\pm \mathrm{SD}, 0.86 \pm 0.079$; range: $0.774-0.951$ ) of those specific brain areas with ALFF differences. Further diagnostic analysis exhibited that those specific areas with ALFF differences alone discriminated the alcohol dependents from the healthy subjects with high degree of sensitivities (mean $\pm \mathrm{SD}, 80.84 \% \pm 14.01 \%$ or $80 \% \pm 14.56 \%$; range: $62.5 \%-100 \%$ ) and specificities (mean $\pm \mathrm{SD}, 83.32 \% \pm 9.31 \%$; range: $70.8 \%-95.8 \%$ or $84.16 \% \pm 8 \%$; range: $75 \%-95.8 \%$ ) (Table 3 and Figure 3 ).

Table 2 The ALFF differences between patients with alcohol dependence and healthy subjects

\begin{tabular}{l|l|l|l|l|l}
\hline Brain regions of peak coordinates & R/L & BA & $\begin{array}{l}\text { Voxel volume } \\
\left(\mathbf{m m}^{3}\right)\end{array}$ & $\begin{array}{l}\boldsymbol{t} \text {-score of } \\
\text { peak voxel }\end{array}$ & MNI coordinates \\
\hline Supplementary motor area & & & $\boldsymbol{X}, \boldsymbol{Y}, \mathbf{Z}$ & $5 \mathrm{I}, 0,5 \mathrm{I}$ \\
Inferior parietal lobule & $\mathrm{R}$ & 6 & 4,509 & 6.2634 & $48,-30,30$ \\
Precuneus & $\mathrm{R}$ & 40 & 3,753 & 3.0616 & $-3,-66,30$ \\
Cerebellum posterior lobe & $\mathrm{L}$ & 7 & 2,133 & -3.2966 & $-24,-81,-36$ \\
Cerebellum posterior lobe & $\mathrm{L}$ & N/A & 2,376 & -4.6716 & $54,-51,-36$ \\
\hline
\end{tabular}

Note: The statistical threshold was set at corrected significance level of individual two-tailed voxel-wise $P<0.05$ using an AlphaSim corrected threshold of cluster $P<0.05$. Abbreviations: BA, Brodmann's area; L, left; MNI, Montreal Neurological Institute; N/A, not applicable; R, right. 


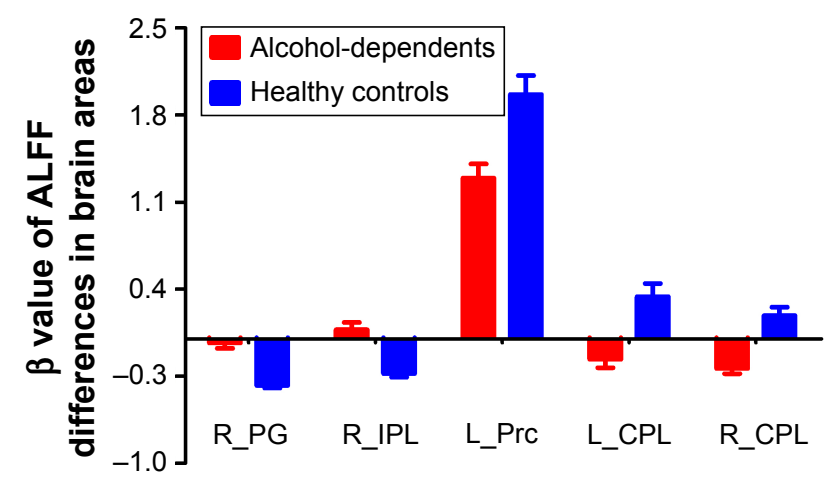

Figure 2 Mean $\beta$ value of ALFF differences in regional brain areas. Abbreviations: ALFF, amplitude of low-frequency fluctuation; CPL, cerebellum posterior lobe; IPL, inferior parietal lobule; L, left; PG, precentral gyrus; Prc, precuneus; R, right.

\section{Pearson correlation analysis}

In the alcohol dependence group, the mean years of alcohol consumption displayed a positive correlation with the $\beta$ value of left precuneus ( $r=0.481, P=0.008$; Figure $4 \mathrm{~A}$ ); other significant correlations between the $\beta$ value of brain areas with ALFF differences and the clinical features were not found $(P>0.05)$. However, several correlations among the $\beta$ value of ALFF differences in brain areas were found (Figure 4B-F). The $\beta$ value of the right supplementary motor area showed a positive correlation with that of the right inferior parietal lobule ( $r=0.497, P=0.006$; Figure $4 \mathrm{~B}$ ), and a negative correlation with that of the left cerebellum posterior lobe ( $r=-0.468, P=0.011$; Figure $4 \mathrm{C}$ ). The $\beta$ value of the left and right cerebellum posterior lobes, respectively, displayed a negative correlation with that of the right inferior parietal lobule (left, $r=-0.649, P<0.001$, Figure 4D; right, $r=-0.56$, $P=0.002$, Figure 4E). The $\beta$ value of the left and right cerebellum posterior lobe also showed a positive correlation with each other $(r=0.373, P=0.046$; Figure $4 \mathrm{~F})$.

\section{Discussion}

The current study is the first to utilize the ALFF analysis to identify altered functional brain areas in 29 patients with alcohol dependence relative to 29 status-matched healthy subjects. ROC curve was applied to identify the ability of those ALFF differences in distinguishing the two groups. The present study revealed the following main results: 1) alcohol dependence was associated with right supplementary motor area and right inferior parietal lobule with significant higher ALFF differences, and left precuneus and bilateral cerebellum posterior lobe with lower ALFF differences; 2) ROC curve revealed good AUC values of those specific brain areas, and further diagnostic analysis demonstrated that those specific brain areas alone discriminated the patients with alcohol dependence from the healthy subjects with high degree of sensitivities and specificities; 3 ) in the alcohol dependence group, the mean years of alcohol consumption displayed a positive correlation with the $\beta$ value of the left precuneus, and several correlations among those specific brain areas were found.

Morphological-anatomical studies have found decreased gray matter volumes in the frontal lobe $\mathrm{e}^{18-21}$ and cerebellum ${ }^{20,22}$ in patients with alcohol dependence. These changes have been shown to be predictive of relapse risk, suggesting a significant role of decreased gray matter volumes in the frontal lobe and cerebellum in clinical outcomes in alcohol dependence. ${ }^{21}$ Similarly, resting-state functional connectivity studies also showed consistent findings. Distinct areas of the cerebellum posterior lobe have shown altered functional brain connectivity with the prefrontal and parietal lobes. ${ }^{23,24}$ Herting et a ${ }^{25}$ found differences of contralateral functional connectivity between the prefrontal cortex and the lateral cerebellum in healthy adolescents, but this brain connectivity is atypical in high risk alcohol-naïve youth subjects with a history of family alcoholism. These disturbed functional connectivities also have been shown in the contralateral frontocerebellar regions, ${ }^{23,24}$ suggesting that white matter fibers decussate and cross over to the contralateral hemisphere between the cerebellum and the cerebral cortex. ${ }^{26}$ The frontocerebellar connectivity was shown to be associated with fractional anisotropy value in the superior longitudinal fasciculus and anterior limb of the internal capsule. Notably, the superior longitudinal fasciculus has projections into the

Table 3 ROC curve for ALFF differences in brain areas between alcohol dependent and healthy subjects

\begin{tabular}{|c|c|c|c|c|}
\hline Brain area & AUC & Sensitivity, \% & Specificity, \% & Cutoff point ${ }^{a}$ \\
\hline R_Supplementary motor area & 0.9510 & $100 \%$ & $79.2 \%$ & -0.3178 \\
\hline R_Inferior parietal lobule & 0.9080 & $87.5 \%$ & $87.5 \%$ & -0.1132 \\
\hline L_Precuneus ${ }^{\#}$ & 0.7800 & $75 \%$ & $70.8 \%$ & 1.5582 \\
\hline L_Precuneus" & 0.7800 & $70.8 \%$ & $75 \%$ & 1.6676 \\
\hline L_Cerebellum posterior lobe & 0.7740 & $62.5 \%$ & $83.3 \%$ & 0.1731 \\
\hline R_Cerebellum posterior lobe & 0.8870 & $79.2 \%$ & $95.8 \%$ & -0.0153 \\
\hline
\end{tabular}

Notes: "The two sets values of L_Precuneus showed the same discriminative abilities. aCutoff point of mean ALFF signal value.

Abbreviations: ALFF, amplitude of low-frequency fluctuation; AUC, area under the curve; L, left; R, right; ROC, receiver operating characteristic. 

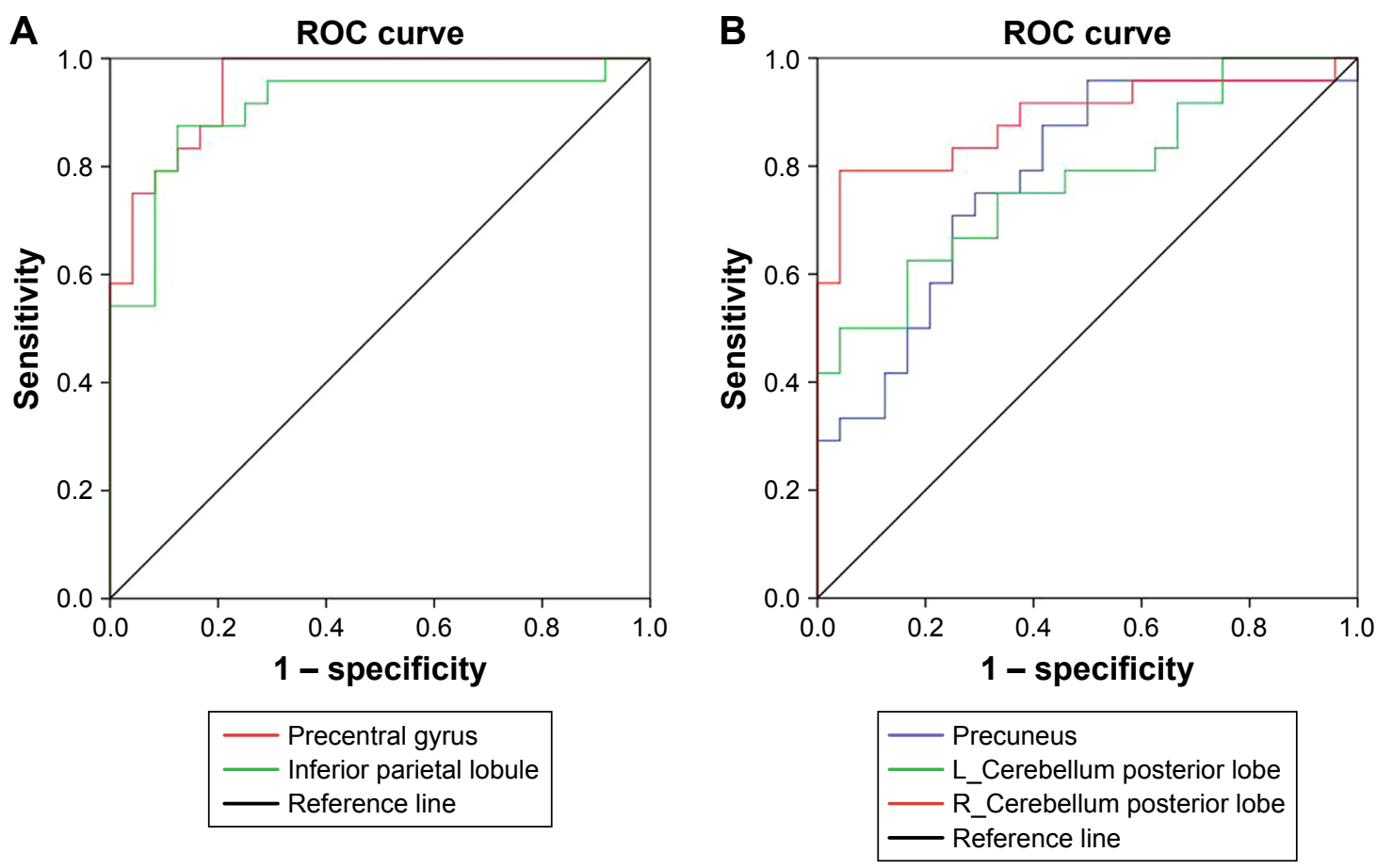

Figure 3 ROC curve analysis of ALFF differences in regional brain areas.

Note: ROC curve of higher (A) and lower (B) ALFF in brain areas.

Abbreviations: ALFF, amplitude of low-frequency fluctuation; L, left; R, right; ROC, receiver operating characteristic.

frontal cortex, while the anterior limb of the internal capsule carries the fibers that project from the frontal lobe to the cerebellum. ${ }^{27}$ These findings showed functional and structural connectivity abnormalities in the prefrontal, parietal, and cerebellar cortex in patients with alcohol dependence. Our results of altered ALFF areas in the prefrontal lobe, inferior parietal lobule, and cerebellum posterior lobe support these findings.
A
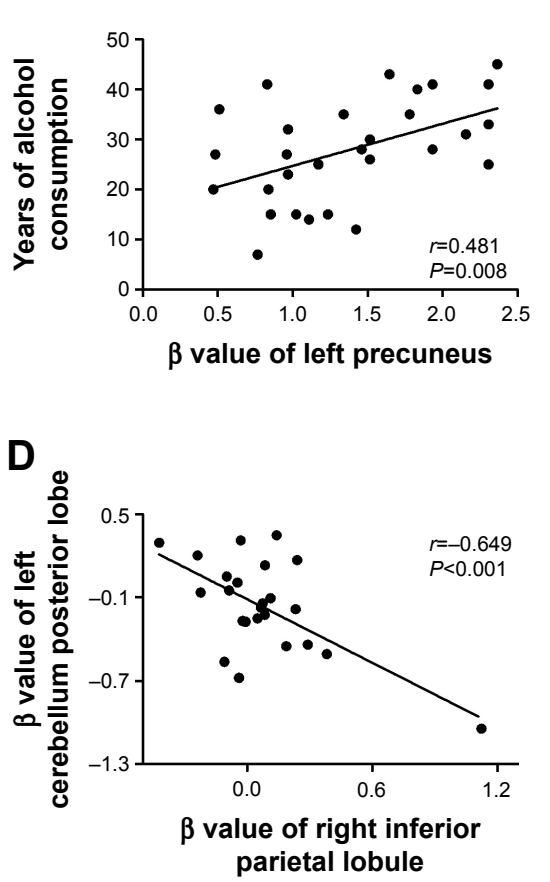

B

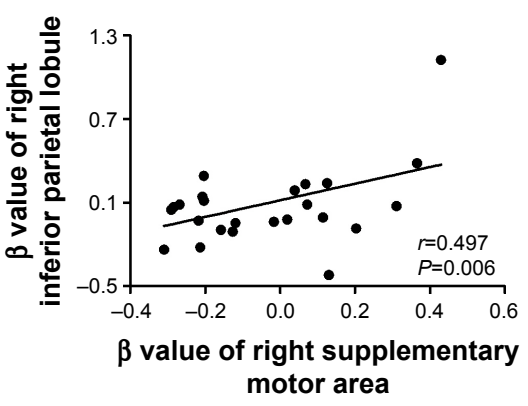

E

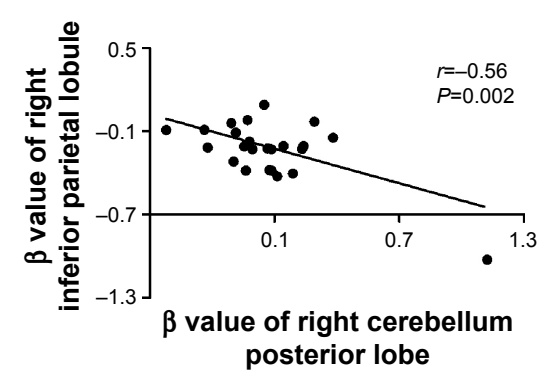

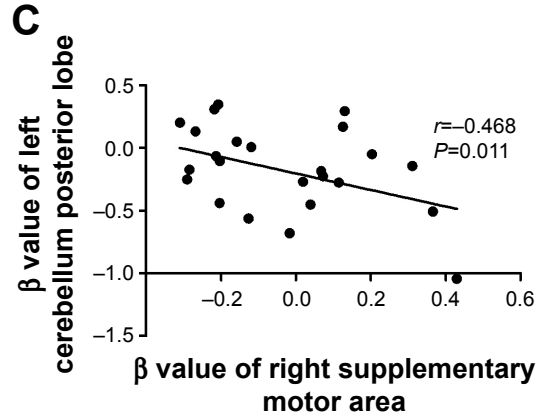

$\mathbf{F}$

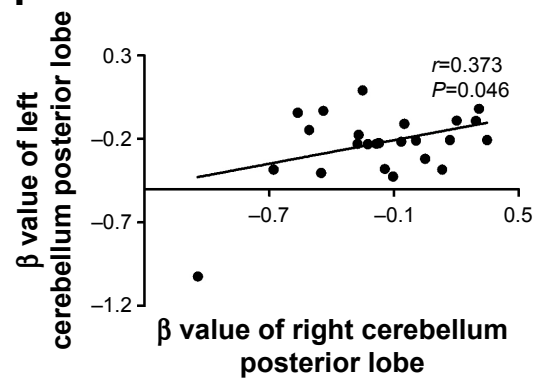

Figure 4 Pearson correlation among characteristics of alcohol dependent and $\beta$ value of ALFF differences in brain areas.

Note: Correlation between years of alcohol consumption and left precuneus (A), and among ALFF differences in brain areas (B-F).

Abbreviation: ALFF, amplitude of low-frequency fluctuation. 
Patients with alcohol dependence had impaired coordination while moving and impaired balance. ${ }^{28}$ Poor regulation of coordinating movement and emotional changes are core characteristics of alcohol dependence. ${ }^{14}$ The cerebellum posterior lobe is associated with regulation of nerve function and coordinating movement, emotion, and cognition, and is particularly vulnerable to alcoholism-related damage. ${ }^{10,28,29}$ The frontal-temporalbasal ganglia and cerebellar circuits have been shown to overlap motor control function and are associated with motor behavior, which is disrupted by alcohol intoxication..$^{15,30}$ The disturbed functional activities in these brain areas may be the main reasons for impaired driving behavior in alcoholics. Our results revealed that the alcohol dependence was associated with altered regional brain activities in the movement-related areas, including the supplementary motor area, cerebellum posterior lobe, and inferior parietal lobule. Furthermore, these movement-related areas were significantly correlated with each other. These areas have been shown to have altered functional connectivity in several studies in patients with alcohol dependence,,$^{11,14,30,31}$ and may therefore support our results.

The precuneus is thought to be engaged in visuospatial imagery, collection and evaluation of information, and self-processing operations, ${ }^{32-34}$ suggesting a key role in the advanced cognitive function. Disturbed regional brain activity or functional connectivity in the precuneus was not only found in primate brain suffer acute exposure to cue of alcohol, ${ }^{35}$ but also in subjects with alcohol use disorder and/ or after heavy drinking. ${ }^{15,36,37}$ The increased brain connectivity between the precuneus and the cerebellum was also found in chronic alcohol consumption..$^{38}$ The metabolic activity in the precuneus was higher, and may require about $35 \%$ glucose, which is more than that needed in other areas. ${ }^{39-41}$ Our data showed that the $\beta$ value of the precuneus with lower ALFF value displayed a positive correlation with mean years of alcohol consumption. The functional brain activity decrease in the precuneus may be interpreted as functional impairment caused by long-term alcoholism.

\section{Conclusion}

In summary, the ALFF analysis may serve as a biological indicator in the detection of regional spontaneous brain activities in patients with alcohol dependence with high degree of sensitivities and specificities. The prefrontalparietal-cerebellar circuit appears to be disturbed by longterm alcoholism in patients with alcohol dependence. The findings of the present study could expand our understanding of the neurobiological mechanisms underlying alcohol dependence, and may help us develop targeted intervention and prevention strategies.

\section{Acknowledgment}

This study was supported by the Key Research and Development Projects in Shaanxi Province in the Field of Social Development (2017SF-081). This was not an industry-supported study.

\section{Disclosure}

The authors report no conflicts of interest in this work.

\section{References}

1. Wise RA. Brain reward circuitry: insights from unsensed incentives. Neuron. 2002;36(2):229-240.

2. Baker TB, Piper ME, McCarthy DE, Majeskie MR, Fiore MC. Addiction motivation reformulated: an affective processing model of negative reinforcement. Psychol Rev. 2004;111(1):33-51.

3. Dai XJ, Liu CL, Zhou RL, et al. Long-term total sleep deprivation decreases the default spontaneous activity and connectivity pattern in healthy male subjects: a resting-state fMRI study. Neuropsychiatr Dis Treat. 2015;11:761-772.

4. Volkow ND, Fowler JS, Wang GJ. The addicted human brain: insights from imaging studies. J Clin Invest. 2003;111(10):1444-1451.

5. Everitt BJ, Belin D, Economidou D, Pelloux Y, Dalley JW, Robbins TW Review. Neural mechanisms underlying the vulnerability to develop compulsive drug-seeking habits and addiction. Philos Trans R Soc Lond B Biol Sci. 2008;363(1507):3125-3135.

6. Koob GF, Volkow ND. Neurocircuitry of addiction. Neuropsychopharmacology. 2010;35(1):217-238.

7. Simpson JR, Snyder AZ, Gusnard DA, Raichle ME. Emotion-induced changes in human medial prefrontal cortex: I. During cognitive task performance. Proc Natl Acad Sci U S A. 2001;98(2):683-687.

8. Mason MF, Norton MI, van Horn JD, Wegner DM, Grafton ST, Macrae CN. Wandering minds: the default network and stimulusindependent thought. Science. 2007;315(5810):393-395.

9. Yan H, Zhang Y, Chen H, Wang Y, Liu Y. Altered effective connectivity of the default mode network in resting-state amnestic type mild cognitive impairment. J Int Neuropsychol Soc. 2013;19(4):400-409.

10. Dai XJ, Nie X, Liu X, et al. Gender differences in regional brain activity in patients with chronic primary insomnia: evidence from a restingstate fMRI study. J Clin Sleep Med. 2016;12(3):363-374.

11. Wang L, Chen Y, Yao Y, Pan Y, Sun Y. Sleep deprivation disturbed regional brain activity in healthy subjects: evidence from a functional magnetic resonance-imaging study. Neuropsychiatr Dis Treat. 2016;12:801-807.

12. Huang X, Zhong YL, Zeng XJ, et al. Disturbed spontaneous brain activity pattern in patients with primary angle-closure glaucoma using amplitude of low-frequency fluctuation: a fMRI study. Neuropsychiatr Dis Treat. 2015;11:1877-1883.

13. Li HJ, Dai XJ, Gong HH, Nie X, Zhang W, Peng DC. Aberrant spontaneous low-frequency brain activity in male patients with severe obstructive sleep apnea revealed by resting-state functional MRI. Neuropsychiatr Dis Treat. 2015;11:207-214.

14. Tu X, Wang J, Liu X, Zheng J. Aberrant regional brain activities in alcohol dependence: a functional magnetic resonance imaging study. Neuropsychiatr Dis Treat. 2018;14:847-853.

15. Luo X, Guo L, Dai XJ, et al. Abnormal intrinsic functional hubs in alcohol dependence: evidence from a voxelwise degree centrality analysis. Neuropsychiatr Dis Treat. 2017;13:2011-2020.

16. Zang YF, He Y, Zhu CZ, et al. Altered baseline brain activity in children with ADHD revealed by resting-state functional MRI. Brain Dev. 2007;29(2):83-91.

17. El Khouli RH, Macura KJ, Barker PB, Habba MR, Jacobs MA, Bluemke DA. Relationship of temporal resolution to diagnostic performance for dynamic contrast enhanced MRI of the breast. J Magn Reson Imaging. 2009;30(5):999-1004.

18. Bühler M, Mann K. Alcohol and the human brain: a systematic review of different neuroimaging methods. Alcohol Clin Exp Res. 2011;35(10): 1771-1793. 
19. Demirakca T, Ende G, Kämmerer N, et al. Effects of alcoholism and continued abstinence on brain volumes in both genders. Alcohol Clin Exp Res. 2011;35(9):1678-1685.

20. Fein G, di Sclafani V, Cardenas VA, Goldmann H, Tolou-Shams M, Meyerhoff DJ. Cortical gray matter loss in treatment-naïve alcohol dependent individuals. Alcohol Clin Exp Res. 2002;26(4):558-564.

21. Rando K, Hong KI, Bhagwagar Z, et al. Association of frontal and posterior cortical gray matter volume with time to alcohol relapse: a prospective study. Am J Psychiatry. 2011;168(2):183-192.

22. Makris N, Oscar-Berman M, Jaffin SK, et al. Decreased volume of the brain reward system in alcoholism. Biol Psychiatry. 2008;64(3): 192-202.

23. Habas C, Kamdar N, Nguyen D, et al. Distinct cerebellar contributions to intrinsic connectivity networks. J Neurosci. 2009;29(26):8586-8594.

24. O'Reilly JX, Beckmann CF, Tomassini V, Ramnani N, JohansenBerg H. Distinct and overlapping functional zones in the cerebellum defined by resting state functional connectivity. Cereb Cortex. 2010; 20(4):953-965.

25. Herting MM, Fair D, Nagel BJ. Altered fronto-cerebellar connectivity in alcohol-naïve youth with a family history of alcoholism. Neuroimage. 2011;54(4):2582-2589.

26. Kelly RM, Strick PL. Cerebellar loops with motor cortex and prefrontal cortex of a nonhuman primate. $J$ Neurosci. 2003;23(23):8432-8444.

27. Wakana S, Jiang H, Nagae-Poetscher LM, van Zijl PC, Mori S. Fiber tract-based atlas of human white matter anatomy. Radiology. 2004; 230(1):77-87.

28. Sullivan EV, Rosenbloom MJ, Pfefferbaum A. Pattern of motor and cognitive deficits in detoxified alcoholic men. Alcohol Clin Exp Res. 2000;24(5):611-621.

29. Dai XJ, Gong HH, Wang YX, et al. Gender differences in brain regional homogeneity of healthy subjects after normal sleep and after sleep deprivation: a resting-state fMRI study. Sleep Med. 2012;13(6):720-727.

30. Rzepecki-Smith CI, Meda SA, Calhoun VD, et al. Disruptions in functional network connectivity during alcohol intoxicated driving. Alcohol Clin Exp Res. 2010;34(3):479-487.
31. Zheng H, Kong L, Chen L, Zhang H, Zheng W. Acute effects of alcohol on the human brain: a resting-state FMRI study. Biomed Res Int. 2015; 2015:947529.

32. Cavanna AE, Trimble MR. The precuneus: a review of its functional anatomy and behavioural correlates. Brain. 2006;129(Pt 3):564-583.

33. Addis DR, Mcintosh AR, Moscovitch M, Crawley AP, Mcandrews MP. Characterizing spatial and temporal features of autobiographical memory retrieval networks: a partial least squares approach. Neuroimage. 2004;23(4):1460-1471.

34. Lundstrom BN, Ingvar M, Petersson KM. The role of precuneus and left inferior frontal cortex during source memory episodic retrieval. Neuroimage. 2005;27(4):824-834.

35. Telesford QK, Laurienti PJ, Friedman DP, Kraft RA, Daunais JB. The effects of alcohol on the nonhuman primate brain: a network science approach to neuroimaging. Alcohol Clin Exp Res. 2013;37(11): 1891-1900.

36. Schacht JP, Anton RF, Myrick H. Functional neuroimaging studies of alcohol cue reactivity: a quantitative meta-analysis and systematic review. Addict Biol. 2013;18(1):121-133.

37. Vergara VM, Liu J, Claus ED, Hutchison K, Calhoun V. Alterations of resting state functional network connectivity in the brain of nicotine and alcohol users. Neuroimage. 2017;151:45-54.

38. Chanraud S, Pitel AL, Pfefferbaum A, Sullivan EV. Disruption of functional connectivity of the default-mode network in alcoholism. Cereb Cortex. 2011;21(10):2272-2281.

39. Fransson P, Marrelec G. The precuneus/posterior cingulate cortex plays a pivotal role in the default mode network: Evidence from a partial correlation network analysis. Neuroimage. 2008;42(3):1178-1184.

40. Raichle ME, Macleod AM, Snyder AZ, Powers WJ, Gusnard DA, Shulman GL. A default mode of brain function. Proc Natl Acad Sci US A. 2001;98(2):676-682.

41. Utevsky AV, Smith DV, Huettel SA. Precuneus is a functional core of the default-mode network. J Neurosci. 2014;34(3):932-940.
Neuropsychiatric Disease and Treatment

\section{Publish your work in this journal}

Neuropsychiatric Disease and Treatment is an international, peerreviewed journal of clinical therapeutics and pharmacology focusing on concise rapid reporting of clinical or pre-clinical studies on a range of neuropsychiatric and neurological disorders. This journal is indexed on PubMed Central, the 'PsycINFO' database and CAS,

\section{Dovepress}

and is the official journal of The International Neuropsychiatric Association (INA). The manuscript management system is completely online and includes a very quick and fair peer-review system, which is all easy to use. Visit http://www.dovepress.com/testimonials.php to read real quotes from published authors. 